\title{
Interference between Foveal and Peripheral Visual Tasks, Potentially Affecting Mesopic Photometry
}

\author{
Yukio AKASHI*, Sueko KANAYA** and Chieko ISHIKURA*** \\ *University of Fukui \\ **Visual Technology Laboratory Inc. \\ ***NEC Lighting, Ltd.
}

Received July 18, 2013, Accepted January 20, 2014

\begin{abstract}
Commission Intemationale de l'Eclairage (CIE) published CIE 191: Recommended System for Mesopic Photometry Based on Visual Performance was published in 2010. However, it is still necessary to identify appropriate applications and make guidelines for the new mesopic photometry system. To this end, we dealt with interference between the foveal task and the peripheral task that often exists in the real driving context. Such interference often appears to exaggerate the effectiveness of short-wavelength radiation on peripheral visual performance at mesopic light levels. To address a question of how the foveal vision affects the peripheral vision for different spectral power distributions, but for the same mesopic luminance, we conducted a controlled laboratory experiment and two supplemental laboratory experiments. In these experiments, we asked subjects to perform a foveal tracking task and a peripheral target-detection task, simulating nighttime multi-tasking driving activities in mesopic light levels. Based on the results of the experiments we confirmed the Purkinje phenomena. We also found interference between the foveal task and the peripheral task (especially when the foveal task was difficult). Such interference not only impairs the foveal task performance but also reduces the peripheral responses significantly, and potentially makes the Purkinje phenomena appear to be exaggerated. This confirmed the importance of using light sources with more short-wavelength radiation for better peripheral visibility in nighttime roadway lighting applications. Such interference also suggests that the use of a simple easy task may help eliminate any effect of interference on databases to establish a unified mesopic photometry system.
\end{abstract}

KEYWORDS: roadway lighting, mesopic vision, mesopic photometry, spectral power distribution, task interference

\section{Introduction}

As is well known, the current photopic photometry system does not consider the Purkinje effect at mesopic light levels in nighttime roadway applications. Recently many attempts have been made to develop performance based mesopic photometry systems bridging the photopic and scotopic luminous efficiency functions ${ }^{122}$. To develop a performance based mesopic photometry, the International Commission on Illumination (CIE) established a technical committee, TC 1-58, in 2002. Among many proposed models for the mesopic vision, the TC 1-58 selected two photometry systems-they were the Unified System of Photometry (USP) and the Mesopic Optimization of Visual Efficiency (MOVE) system. The USP was based on subjects' responses against high luminance contrast targets in order to extract visual performance solely related to the achromatic channel. The MOVE system, on the other hand, was based on subjects' responses to targets with a wide range of luminance contrasts as well as subjects' increment thresholds. After constructive discussions, the TC 1-58 reached a consensus of a compromised system (MES2) between the two systems in $2010^{3)}$. However, it is still necessary to understand how to apply the MES2 system for the real world. The CIE's JTC-01 currently has worked on this problem.

It is obvious that the use of these mesopic photometry systems results in much better agreements with true visual performance at mesopic light levels than the use of the current photopic photometry system based on $V(\lambda)$. However, the mesopic photometry systems cannot simply replace the current photopic photometry system. The mesopic photometry systems serve to represent the stimulus for visual performance experiments under different light levels and spectral power distributions (SPDs) in the peripheral visual field, but not necessarily in the central (foveal) visual field. The mesopic photometry systems are responsible for visual 
tasks in the peripheral visual field. Recent studies suggested that the visual performance in reaction times for small foveal stimuli changes little with SPD and is described adequately by $V(\lambda)^{1 / 4}$. In addition, there may be interference between the foveal task and the peripheral task in the real driving context. Such interference often appears to exaggerate the effectiveness of shortwavelength radiation on peripheral visual performance at mesopic light levels ${ }^{5)-7}$. A common question about the use of the mesopic photometry systems for specifying light in nighttime applications is how the foveal vision affects the peripheral vision for different SPDs, but for the same mesopic luminance (where both rods and cones contribute to the visual system in the periphery). If any interference between foveal tasks and peripheral tasks in the experiments, from which the mesopic photometry was derived, exists, it may have affected the accuracy of the mesopic photometry. On the other hand, when lighting specifiers apply the mesopic photometry for nighttime roadway applications where any foveal-peripheral task interference occurs, they can expect a somewhat larger Purkinje effect than the mesopic photometry indicates.

To address the above-described question, we conducted a controlled laboratory experiment and two supplemental laboratory experiments. In the main experiment, we asked subjects to perform a foveal tracking task and a peripheral target-detection task simultaneously, simulating nighttime multi-tasking driving activities in mesopic light levels-we call this experiment as "a dual task experiment." The results of this experiment allowed us to understand whether there is interference between the foveal task and peripheral task. In one of the two supplemental experiments, subjects did not perform a foveal task but a peripheral task-"a single task experiment". The results of this experiment allowed us to confirm the results of the dual task experiment. In the second supplemental experiment, subjects conducted the same foveal and peripheral tasks as the dual task experiment but at a much higher photopic light level to understand the best performance of the subjects- "a photopic task experiment".

\section{Experiments}

First, the dual task experiment took place in a laboratory on one day, and then the other two experiments took place later together in the same laboratory on another day. In the dual task experiment, 15 subjects $(8$ males and 7 females, 23 years old on the average) participated. Among the 15 subjects, six subjects participated in the two supplemental experiments.

\subsection{Light sources and lighting conditions}

We designed these experiments in order to address the effect of foveal task load on peripheral detection by comparing two light sources, widely used for street lighting applications in Japan. These light sources were a high-pressure sodium lamp (HPS) and a fluorescent lamp (FL). Figure 1 depicts the spectral power distributions of the lamps. The HPS has an $\mathrm{S} / \mathrm{P}$ ratio of 0.44 while 1.97 for the FL. The $\mathrm{S} / \mathrm{P}$ ratio is defined as the ratio of scotopic luminance against photopic luminance. This means that the FL emits more short-wavelength radiation than the HPS lamp does, creating a more effective stimulus for the peripheral vision at the same photopic light level.

By using these two lamps, three lighting conditions were prepared as shown in Table 1 for the dual task experiment. These three conditions were determined by using the USP (Rea et al., 2004). This was because the USP is solely based on reaction times of subjects' target detections. The procedure of the USP calculation is described in Appendix. Our experiment also analyzed reaction times of subjects' responses to peripheral target presentations as described in the first section. Table 1 shows the experimental conditions. The first condition used HPS, simulating a typical roadway lighting condition at a nighttime mesopic light level. The second condition provided the same photopic luminance for the central and peripheral targets as the HPS condition (fluorescent high or FLH). The third condition provided a lower photopic luminance but the same unified luminance (in terms of USP) as HPS did (fluorescent low or FLL).

On the other hand, the photopic task experiment

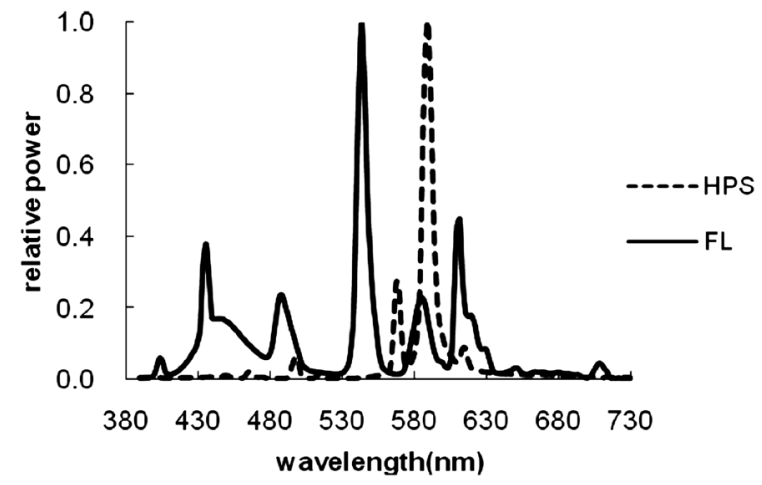

Figure 1 Spectral power distributions of lamps used in the experiment

Table 1 Lighting conditions in the dual task experiment

\begin{tabular}{c|c|c}
\hline Lighting condition & $\begin{array}{c}\text { Photopic } \\
\text { luminance }\left(\mathrm{cd} / \mathrm{m}^{2}\right)\end{array}$ & $\begin{array}{c}\text { Unified } \\
\text { luminance }\left(\mathrm{cd} / \mathrm{m}^{2}\right)\end{array}$ \\
\hline HPS & 0.10 & 0.06 \\
FLH & 0.10 & 0.15 \\
FLL & 0.03 & 0.06 \\
\hline
\end{tabular}


provided a much higher luminance levels than the above-described three lighting conditions by using the same FL as the other experiments.

\subsection{Experimental setup}

We attempted to prepare an experimental setup that produces a simplified nighttime driving context in which a driver conducts a tracking task in the foveal vision and a peripheral detection task in the peripheral vision.

The tracking task simulated a visual task in which a driver turns a steering wheel to manipulate a car, which tends to sway due to the inclination and roughness of the roadway surface, in order to maintain the car within a traffic lane.

The detection task simulated a visual task in which a driver detects peripheral potential hazards such as bicycles on the road shoulder, pedestrians on the pavement, and animals passing across the forward roadway in order to avoid collisions with them.

Such a simulation should simulate a driving context as practical as possible to mimic a subject's real mental task load. However, a practical experimental context often results in noisy data including influences of numerous extraneous variables as seen in many field studies.

Therefore, we attempted to eliminate such extraneous variables by employing a dual task paradigm as a controlled laboratory experiment that is often employed in studies on "attention" in the field of cognitive science. In this effort, we simplified the visual tasks by selecting a uniform background and high contrast detection targets. Additionally, we attempted to minimize a subject's motor response component in response time by selecting quicker actions than real operations while maintaining the subject's visual response and cognitive response components similar to a real driving task. For the peripheral detection task, a subject responded to stimulus by releasing a manual switch by the left index finger instead of stepping on the break. For the central tracking task, a subject rotated a small knob by the right index finger and the thumb instead of controlling a wheel by the hands.

All the three experiments used a single experimental setup composed of a partition, a tracking apparatus, four peripheral targets, a manual switch to signal target detection, a control knob for the tracking task, and a personal computer system to control the peripheral targets and the tracking task, and record subjects' responses. Figure 2 depicts the experimental setup. The experimental setup was located in a $5 \mathrm{~m}$ width, $8 \mathrm{~m}$ length, and $3 \mathrm{~m}$ height laboratory. The ceiling, walls, and floor of the laboratory were painted matt black.

Each of the FL and HPS luminaires was placed on a $0.7 \mathrm{~m}$ high desk at a distance of $3 \mathrm{~m}$ from the partition

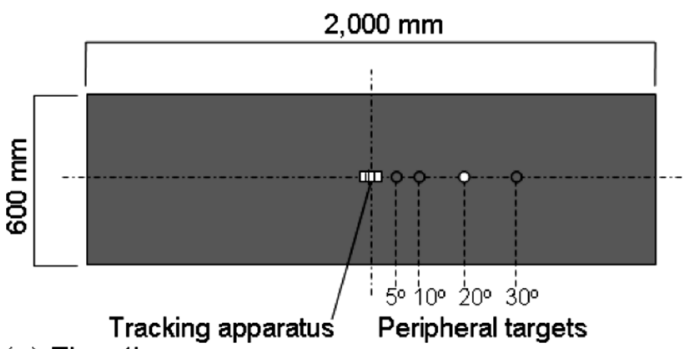

(a) Elevation

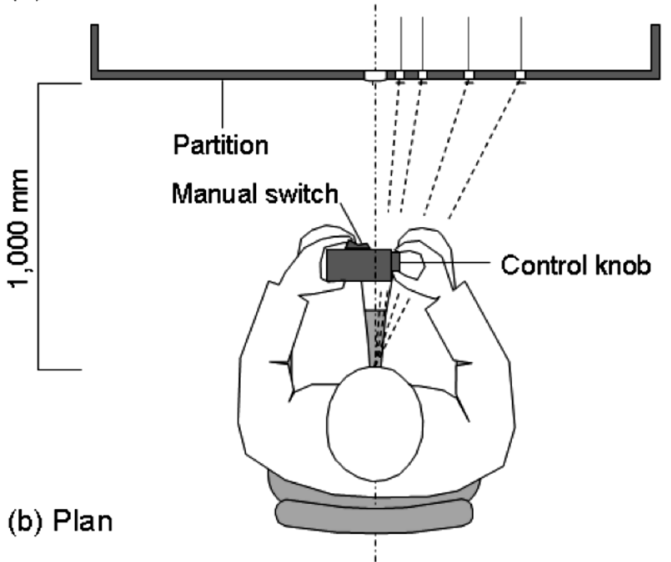

Figure 2 Diagram of experimental setup

(behind the subject) and was aligned with the center of the partition and the center of the subject chair. The luminaires provided upward light to the matt black ceiling. Light reflected on the ceiling indirectly illuminated the experimental setup uniformly. The FL luminaire was a $1.22 \mathrm{~m} \times 0.61 \mathrm{~m}$ rectangular luminaire with two $32 \mathrm{~W}$ FL lamps while the HPS luminaire was a $0.61 \mathrm{~m} \times$ $0.61 \mathrm{~m}$ square luminaire with a $100 \mathrm{~W}$ HPS lamp. Each luminaire had an acrylic lens diffusing light emitted by the lamps. Since the acrylic lenses slightly changed the SPDs of the lamps, we measured the SPDs of light coming from the lenses (Figure 1). A black aluminum foil with a $0.60 \mathrm{~m} \times 0.60 \mathrm{~m}$ opening covered each luminaire lens so that the two luminaries could provide similar Lambertian luminous distributions. We dimmed the luminaries by using black mesh filters over the openings of the black aluminum foils to achieve each lighting condition without changing the SPDs of the luminaires.

The partition was $2 \mathrm{~m}$ wide and $0.6 \mathrm{~m}$ high with a reflectance of $5 \%$. The partition was placed on a $1 \mathrm{~m}$ high table. In the center of the partition, the foveal tracking task apparatus was located to direct subject fixation on the central visual field. The foveal tracking task apparatus was composed of a $10 \mathrm{~mm}$ high and $40 \mathrm{~mm}$ wide rectangular background and a black $20 \mathrm{~mm}$ long and $2 \mathrm{~mm}$ wide needle. These dimensions for the immediate background were chosen so that the apparent visual size of the immediate background can be covered roughly by the 2-degree foveal visual field. In this way, a subject could perform the foveal task with- 
out moving the eyes widely.

The black needle is a needle of a voltage meter of which location can be adjusted by the voltage applied to it. By changing the voltage in random direction (positive or negative) every $10 \mathrm{~ms}$, the black needle was moved from side to side randomly over the background. By rotating the control knob, a subject attempted to change the voltage applied to the voltage meter so that the subject can maintain the position of the needle in the center of the immediate background. In order to change the luminance contrast of the needle against the background and therefore change the difficulty of the foveal task, the reflectance of the background was changed in two conditions, i.e., $83.7 \%$ for a high reflectance and $32.8 \%$ for a low reflectance. As for the needle, the reflectance was constant at $7.4 \%$. Therefore, the luminance contrast of the needle against the immediate background was 0.91 for the higher reflectance of the immediate background while 0.77 for the lower reflectance.

In the right side of the foveal target, four peripheral detection targets were placed at the same height as the foveal target. Each detection target was a $13 \mathrm{~mm}$ diameter disk and was flipped electromagnetically from a black side (reflectance: 5\%) to a white side (reflectance: $80 \%)$. The contrast of the white side to the background, i.e., the partition was 15. Each of the four targets was presented seven times under each lighting condition and for each of the reflectance levels for the tracking task.

The manual switch and control knob were equipped together with a small black plastic box for a subject to hold in his/her hands during target detections. The subject rotated the control knob by the right hand while depressing and releasing the manual switch by the left hand.

\subsection{Experimental design and conditions}

Independent variables in the dual task experiment were the lighting condition, the luminance contrast of the foveal target, and the eccentricity angle of the offaxis target. Table 2 shows the experimental conditions for these variables. There were 24 (three lighting conditions $*$ two contrasts $*$ four target positions) conditions. Each condition was repeated seven times so there were 168 presentations in total in each session.

The idea behind this experimental design was to set different mental task load levels (i.e., difficulty levels), associated with the tracking task. When the luminance contrast of the needle against its background was lower, the mental task load was supposed to be higher than when the luminance contrast was higher. To confirm this tendency, as the lowest mental task load, we conducted the single task experiment. In the single task experiment, subjects responded to the peripheral
Table 2 Experimental conditions

\begin{tabular}{lc}
\hline Independent variables & Levels \\
\hline Lighting condition & HPS, FLL, FLH \\
Foveal target contrast against background & High, Low \\
Eccentricity angle from the center (degrees) & $5,10,20,30$ \\
\hline
\end{tabular}

Table 3 Summary and abbreviations of conditions in three experiments

\begin{tabular}{|c|c|c|c|c|}
\hline Experiment & Abbreviated & Lamp SPD & $\begin{array}{l}\text { Photopic } \\
\text { luminance }\end{array}$ & $\begin{array}{c}\text { Luminance } \\
\text { contrast }\end{array}$ \\
\hline \multirow{6}{*}{$\begin{array}{l}\text { Dual task } \\
\text { experiment }\end{array}$} & HPS_H & HPS & High & High \\
\hline & HPS_L & HPS & High & Low \\
\hline & FLH_H & FL & High & High \\
\hline & FLH_L & $\mathrm{FL}$ & High & Low \\
\hline & FLL_H & $\mathrm{FL}$ & Low & High \\
\hline & FLL_L & $\mathrm{FL}$ & Low & Low \\
\hline \multirow{3}{*}{$\begin{array}{l}\text { Single task } \\
\text { experiment }\end{array}$} & HPS_N & HPS & High & None \\
\hline & FLH_N & FL & High & None \\
\hline & FLL_N & $\mathrm{FL}$ & Low & None \\
\hline \multirow{2}{*}{$\begin{array}{l}\text { Photopic } \\
\text { experiment }\end{array}$} & FLP_H & $\mathrm{FL}$ & Highest & High \\
\hline & FLP_L & $\mathrm{FL}$ & Highest & Low \\
\hline
\end{tabular}

targets without the foveal tracking task. By comparing reaction times between the dual task experiment and the single task experiment, it is possible to confirm the interference between the foveal task and the peripheral task influencing peripheral reaction times. There were 84 (three lighting conditions $*$ four target positions $*$ seven repetitions) presentations in total.

In order to understand the best performance under a bright condition, we conducted the photopic experiment by using the same fluorescent lamps as the one used in the dual task experiment. In this experiment, the luminance of the peripheral targets was $36.8 \mathrm{~cd} / \mathrm{m}^{2}$. The use of such a photopic task may help scale the effect of lamp SPDs on performance shown in the dual task experiment by understanding the best performance at a much brighter photopic light level. In the photopic task experiment, 56 (two foveal tasks $*$ four target positions $*$ seven repetitions) conditions were presented. Table 3 summarizes experimental design and abbreviations of experimental conditions.

When setting lighting conditions during the experiment, we measured illuminances at fixed five points along the horizontal line with a height of $0.3 \mathrm{~m}$ from the table surface on the partition. Those five points were located at eccentricity angles of $0,10,20$, and 30 degrees from the center to right, and 30 degrees to left. 
We adjusted the position and orientation of each luminaire until all six measured illuminances became the same as each of the target illuminances so that the illuminance distribution on the partition could be uniform in the entire visual field. When we measured illuminances over the partition after the experiment, however, an illuminance at the edge of the partition (at an eccentricity angle of about 50 degrees) was approximately $10 \%$ lower than illuminance in the center.

\subsection{Experimental procedure}

In the dual task experiment, an experimenter escorted a subject to the laboratory and seated the subject on a chair in the laboratory where one of the lighting conditions had been already presented. The height of the chair was changed so the subject's eye height was the same as the height of the foveal task. Prior to the experiment, the subject practiced the tasks for more than 5 min while adapting to the lighting condition. After the practice, the subject started the first dual task experiment combining the foveal tracking task and the peripheral detection task under one of the three lighting conditions.

The foveal tracking task was to keep the needle in the display's center by rotating a control knob. The peripheral task was to detect one of the four targets flipped from the black side to the white side and to release a switch immediately upon detecting the target presentation for signaling the target detections. Then, the period between when the peripheral target was presented and when a subject released the switch was recorded as a reaction time under each of the experimental conditions. To measure and record the performance of the tracking task, the position of the needle was monitored 100 times per a second throughout each trial by measuring the distance of the needle from the center of its background; smaller deviations from the center indicate better foveal visual performance. As stated above, subjects performed the foveal task and the peripheral target detection task simultaneously.

After the above-described experiments, six of the 15 subjects conducted the single task experiment and the photopic task experiment. These experiments followed the same procedure as the dual task experiment. The difference of the single task experiment from the dual task experiment was that subjects simply performed peripheral detections without any foveal tasks. In the photopic task experiment, subjects performed both the foveal task and peripheral task at a time but at a photopic light level.

\subsection{Results}

\subsubsection{Peripheral reaction times in dual task experi- ment}

By using reaction time data collected in the dual task experiment, a mean value and a standard deviation were calculated for each of the four peripheral targets under each of the six experimental conditions (i.e., three lighting conditions * two foveal task conditions). Reaction times over $800 \mathrm{~ms}$ were excluded from the calculation data and such trials were regarded as missed trials. Based on the criterion, we identified 30 missed trials in total in the dual task experiment. Figure 3 shows the results of the calculations. To statistically confirm tendencies shown in Figure 3, a three-way analysis of variance (ANOVA) was applied to all the 2520 (six experimental conditions $*$ four target positions $*$ seven repetitions $* 15$ subjects) reaction times. In this ANOVA, missed trials were treated as missing data.

Since a large number of statistical tests are done and therefore the possibility of a type I error (false positives) is increased, it is better when examining the experimental data to place greater value on a higher level of statistical significance (i.e., $p<0.01$ rather than $p<0.05$ ). Experimental results and interpretation, which meet this criterion, are listed below. The ANOVA revealed significant main effects of target position (as seen in Figure 3) and foveal target contrast (Figure 4) and a significant interaction between lighting condition and target contrast (Figure 4).

To compare reaction times among the four target positions, additional $t$-tests were conducted. The results of the $t$-tests suggest that reaction times for the 5 - and 10-degree targets were shorter than for the 20- and 30 -degree targets $(p<0.001)$. The reaction times of the 30 -degree target were the longest $(p<0.01)$. Such tendencies regarding target position are discussed later in the discussion section.

To analyze the above-described main effects and interaction further, $t$-tests were applied to all the combinations among the six experimental conditions. The

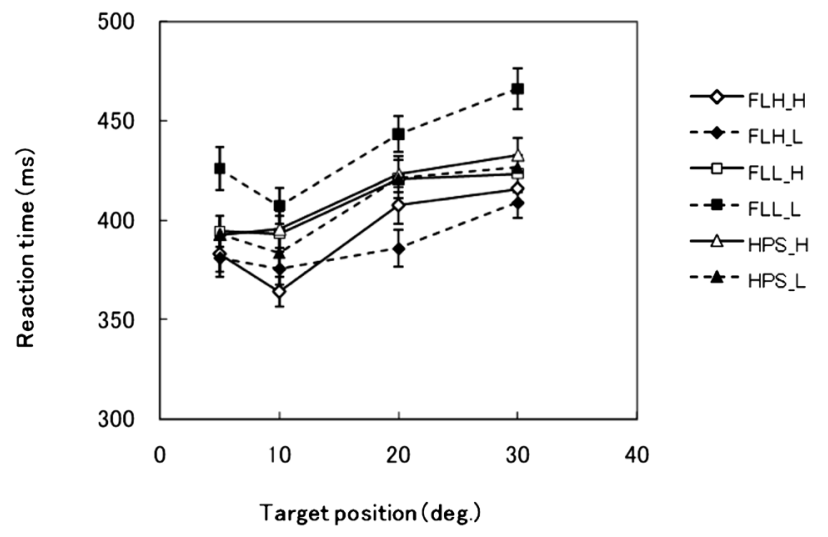

Figure 3 Visual performances for the foveal task and the peripheral detection task. Also shown are standard errors under different lighting condition. Reaction times and needle eccentricity distances were averaged across all subjects. 


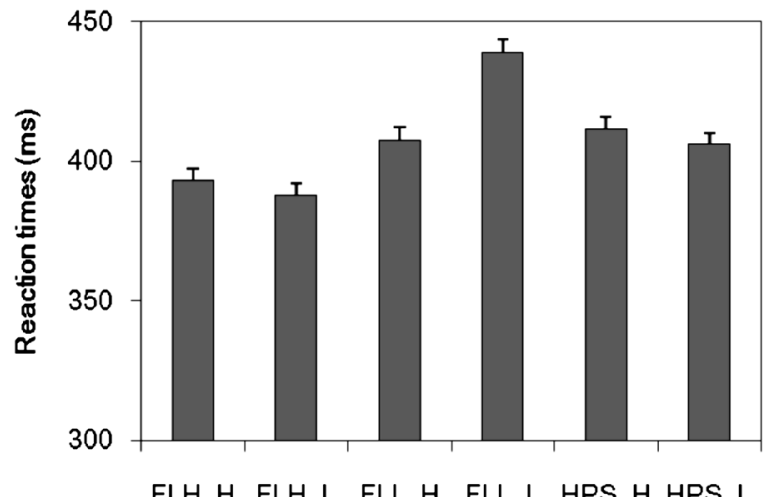

Lighting and contrast conditions

Figure 4 Reaction times for different lighting and contrast conditions

results of the $t$-tests revealed the following tendencies. (1) The effect of foveal target contrast on peripheral visual performance was negligible within each of FLH and HPS. This sounds natural because the unified luminances of the peripheral targets were identical between FLH_H and FLH_L and between HPS_H and HPS_L. Although the luminance contrasts of the central target against the background were different between each of the pairs, it is unlikely that the difficulty of the foveal task affects peripheral task performance. For FLL, however, foveal target contrast does have a significant influence on peripheral task performance $(p<0.001)$. This reflects the above described significant interference between the foveal and the peripheral tasks. This interference will be discussed later in Section 3 .

(2) There were no differences in peripheral performance among FLL_H, HPS_H, and HPS_L. This is natural since unified luminances of peripheral targets were identical under these three conditions. Although the photopic luminance of the foveal task under FLL_H was lower than under HPS_H and HPS_L, the foveal task contrast under FLL_H might be still high enough to maintain peripheral task performance.

(3) Under FLH_H $(p<0.01)$ and FLH_L $(p<0.001)$, peripheral performances were better than under the other experimental conditions. This is probably because mesopic luminances of peripheral targets under FLH_H and FLH_L were higher than the other four conditions.

\subsubsection{Foveal task performance in dual task experi- ment}

Figure 5 shows the results of the foveal performances for the six experimental conditions in the dual task experiment. A mean value and a standard deviation for the subjects' reaction times were obtained for each of the six combinations (i.e., three lighting conditions * three foveal task conditions). To confirm tendencies

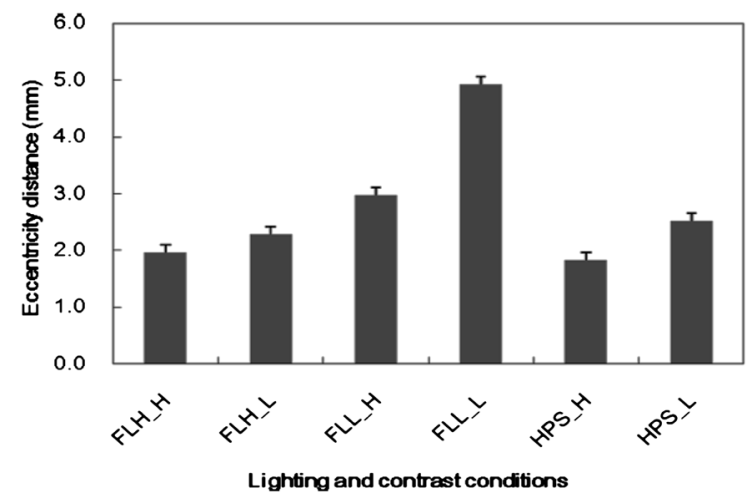

Figure 5 Eccentricity distances for foveal task under different lighting and contrast conditions

shown in Figure 5 statistically, a three way ANOVA was conducted. The ANOVA revealed significant main effects of lighting condition and luminance contrast, and a significant interaction between lighting condition and contrast. It is natural that the target position did not have a main effect for the foveal performance. To analyze the effects and the interaction further, $t$-tests were applied to each of all the 15 pairs among the six conditions. All the above-described analyses suggest the following results.

The results of the $t$-tests showed that the foveal performance under FLH was the same as under HPS. The foveal performance under FLL was worse than under FLH and HPS $(p<0.001)$. This reflects the fact that photopic luminances for the foveal target under FLH and HPS were identical and they were higher than under FLL.

The $t$-test revealed significant effect of luminance contrast under FLL and HPS $(p<0.001)$. As the luminance contrast of the foveal target against the background increases, the foveal performance may be also improved. However, there were no significant differences in performance between the two contrasts under FLH. This may be because foveal performances under both FLH_H and FLH_L were high enough to maintain a high performance level. The significant interaction between lighting condition and contrast suggests that the foveal task may be at the threshold where half of the subjects could not see the foveal needle.

\subsubsection{Results of single task experiment}

To confirm the results of the dual task experiment we conducted the single task (the peripheral task without the foveal task) experiment under FLH, FLL, and HPS conditions by using six of the 15 subjects. From the experimental data, we calculated a mean reaction time for each target position for each lighting condition. Figure 6 shows the results of the reaction times for different target positions under the three 


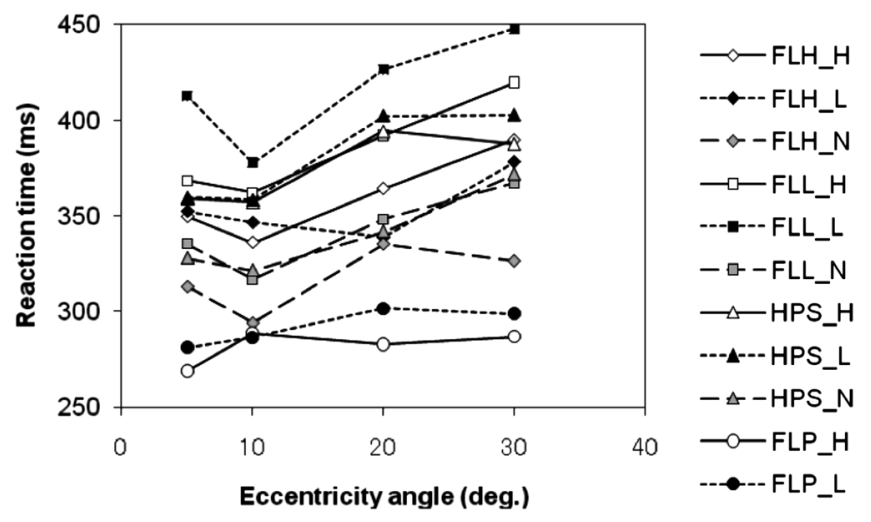

Figure 6 Reaction times for peripheral task under different lighting conditions. This figure depicts six of the 15 subject data.

lighting conditions in the single task experiment with those under six lighting conditions in the dual task experiment. The mean reaction times in the dual task experiment were derived from the six subjects who conducted all the nine experimental conditions. Figure 6 also shows the results of the photopic experiment, which are described in the next section.

To analyze tendencies shown in Figure 6 statistically, we applied a three-way ANOVA to all the nine experimental conditions by using the six subjects' data. The ANOVA revealed significant main effects of target position, lighting condition, and luminance contrast as well as an interaction between lighting condition and luminance contrast $(p<0.01)$. The results of the ANOVA confirmed the tendencies already found in Figure 3. To compare reaction times between with- and withoutfoveal task conditions, we applied $t$-tests to combinations between FLH_H and FLH_N, between FLL_H and FLL_N, and between HPS_H and HPS_N. The $t$ tests revealed significant differences for all the combinations $(p<0.01)$. This confirms that as foveal task load decreases, peripheral performance improves.

In order to confirm the interference between the foveal task and the peripheral task, we applied a three way ANOVA to the six subjects' reaction times for the three factors, i.e., target position, foveal task position, and lamp SPD. The results of the ANOVA showed main effects for all the three factors $(p<0.01)$. However, there was no significant interaction for the combination of the foveal task position and lamp SPD. This means that the task load assigned to the foveal task was not heavy enough to interfere the peripheral task.

Between HPS_N and FLL_N, which have the same mesopic luminance (in terms of USP), there was no significant difference in reaction time. This is reasonable because these two conditions did not have any foveal tasks. There was no significant difference between HPS_L and FLL_L either. This means that the foveal task interferes to the peripheral task.

\subsubsection{Results of photopic task experiment}

To better understand visual performance at a photopic condition, the photopic experiment was conducted. The purpose of this experiment was to understand the best performance at a brighter photopic light level so we can scale how large the effects of SPD on reaction times compared to the effects of illuminance. Figure 6 also includes mean reaction times for each of the eccentricity angles under FLP_H and FLP_L. To understand the effects of photopic light levels on peripheral performance, we applied $t$-tests to a pair of FLH_H and FLP_H and a pair of FLH_L and FLP_L. The $t$-tests revealed significant differences in reaction time in each of the pairs $(\phi<0.01)$. In contrast, there were no significant differences between FLP_H and FLP_L, suggesting little influence of foveal target contrast on peripheral performance. Interestingly, the mean reaction time responding to the five-degree target was the shortest in each of the two experimental conditions while the mean reaction time to the 10-degree target was the shortest at mesopic light levels. This is because only cones contribute to the peripheral target performance at the photopic light level while rods join the contribution to the vision at the mesopic light levels.

\section{Discussion}

\subsection{Interference between foveal and peripheral tasks}

The above-described experimental results suggest that interference occurs between the foveal task and the peripheral task, whereby the difficulty encountered in performing the tracking task under the lower photopic light level in the FLL condition could have made it more difficult to respond to the peripheral targets than in the HPS condition, which had a higher photopic light level. Such interference might prevent drivers from allocating enough attention to both tasks and therefore could result in lower performance of both foveal and peripheral tasks.

To discuss the mechanisms of the above-described interference between the foveal and peripheral tasks, we compared our experimental results with fundamental findings in the field of cognitive science as follows ${ }^{8)}$.

Driving is a demanding, multitasking activity. A driver has to maintain a vehicle within a traffic lane while keeping its speed below the speed limit, while following traffic streams. This task can be regarded as a "tracking task". On more critical occasions the driver must quickly detect oncoming traffic and obstacles on the road shoulder, to avoid collisions with these potential hazards-a "detecting task". As task load increases, a driver, who is already busy with tracking task, can become distracted from the detection tasks. High task 
loads often prevent drivers from detecting potential hazards promptly ${ }^{910)}$.

For each of the tasks, a driver must deal with a sequence of three distinctive information-processing stages: perceptional analysis (stimulus identification); response selection (decision-making); and motor response (response execution) ${ }^{1112)}$. These stages are often thought to occur sequentially, so the results of one stage form the next input. Among them, response selection (decision-making) is the most demanding stage for the brain. This stage limits the use of attention resources that can be shared with the other tasks, and it often appears to act as a bottleneck ${ }^{13)}$.

Recent studies have attempted to reveal the mechanisms of dual-task performance. The time it takes to respond to the second stimulus generally becomes longer than when the second stimulus is presented alone or the task becomes more demanding. This is because the decision-making stage of the second stimulus cannot begin until the corresponding stage of the first stimulus has been completed ${ }^{11)}$. The decision-making stage of the first stimulus can share attention resources with that of the second stimulus when both tasks are very difficult ${ }^{14}$. In these cases, a kind of interference occurs, and the decision-making of the first stimulus is often slowed down as well.

By following the mechanisms of information processing stages, we considered what was happening in the brain of a subject in the dual task context. In the foveal task, when the needle moves away from the center, the subject attempts to return the needle by rotating the knob. When no foveal task is assigned or an easy foveal task is assigned, the subject can respond to the peripheral targets while handling the foveal task. When the foveal task is difficult, the subject took more time to perform it. Then, when an off-axis target is presented, the subject needs more time to detect the target because the subject can start the target detection after finishing the decision making stage of the foveal task. Additionally, when the peripheral target is difficult for the subject to detect, the subject delays responding to the peripheral target since the subject first have to deal with the next move of the needle. Therefore, difference in reaction time of the peripheral tasks between the FL and the HPS appears to increase.

Such potential mechanisms imply that for establishing a photometry system, it is important to reduce the difficulty level of the foveal task and therefore minimize the interference between the foveal and peripheral tasks. When implementing the unified photometry to the real world, on the other hand, we should remember that the use of light sources with more short-wavelength radiations than conventional light sources is important for better peripheral visibility. Importantly, when we use such light sources, we should not reduce illuminance levels to prevent foveal tasks from becoming more difficult and impairing the peripheral task performance as well.

As described in Section 2.2, we employed simplified visual tasks. For the peripheral detection task, a subject responded to stimulus by releasing a manual switch by the left index finger instead of stepping on the break. For the central tracking task, a subject rotated a small knob by the right index finger and the thumb instead of controlling a wheel by the arms. This was because we wanted to minimize a subject's motor component while maintaining visual and cognitive components in order to eliminate the effect of extraneous variables on the subject's responses.

We believe that the above-described strategy worked well, and therefore the results showed clear tendencies. This is why we could find interference between the foveal task and the peripheral task. However, since we employed visual tasks simpler and easier than the real tasks, the results and findings may not represent the real driving context. It is important to employ realistic visual tasks before implementing our findings to the real world.

\subsection{Target positions}

Both USP and MOVE are based on visual performance at a single target eccentricity angle of 15 degrees where the density of rods in the retina is the maximum. The density of cones steeply decreases from the fovea to the periphery at an eccentricity angle around 5 degrees. In contrast, the density of rods is zero at the fovea, while steeply increasing as the eccentricity angle increases, and reaching its peak around 15 degrees ${ }^{15}$. It is reasonable for an international mesopic photometry system to define a single visual angle as its standard condition. However, as shown in Figures 3 and 6, there are significant differences in reaction time among the target positions.

When implementing the mesopic photometry system in roadway and vehicle lighting applications, such angular differences in reaction time may affect the evaluations on the effectiveness of lamp SPDs on visual performance. It may be the case when an appropriate distribution of lamp SPDs is determined for vehicle forward lighting. In such a case, a proper lamp SPD can be adjusted to the characteristics of the retinal spectral sensitivity differently depending on the eccentricity angle. By using different photometry systems for each of the eccentricity angles from the central visual field, the accuracy of forward lighting design and therefore the effectiveness of industrial products can be improved.

\subsection{Comparison between MOVE and USP}

The experimental conditions in this paper were selected according to the USP model. This is simply 
because that the USP model is purely based on reaction times from a high-contrast simple visual task. The counterpart, the MOVE model, is also based on visual performance, but both reaction time tasks with various luminance contrasts and luminance increment tasks. To show the difference of the MOVE model from the USP model in reaction time, we calculated mesopic luminances by using the MOVE system and the USP.

Figures 7 (a) and 7 (b) shows relationships between mesopic luminance and reaction time for our experimental conditions, comparing the two mesopic photometry systems. Both Figures 7 (a) and 7 (b) suggest good correlations between mesopic luminance and reaction time under the easy task and the easiest task conditions. Additionally, the correlation of the USP appears to be better than the correlation of the MOVE system. Again, this may be because the MOVE system was derived from not only reaction time but also other performance data while we measured only reaction times in our experiments. More importantly, under the difficult task condition with the lower (or lowest) mesopic luminance, subject's reaction times show a larger

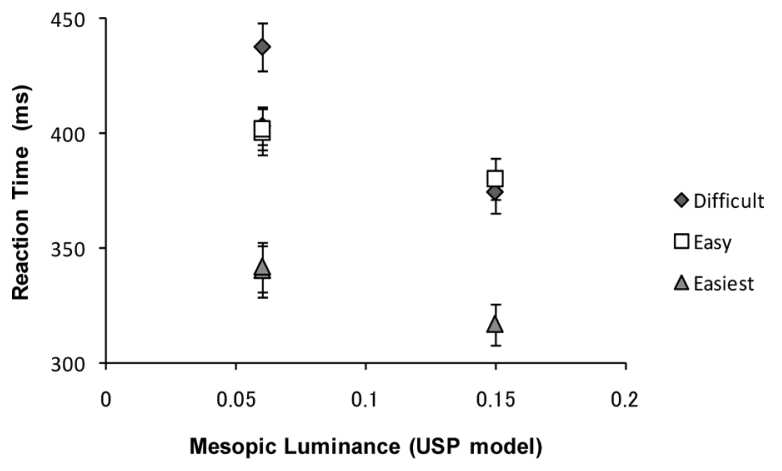

(a)

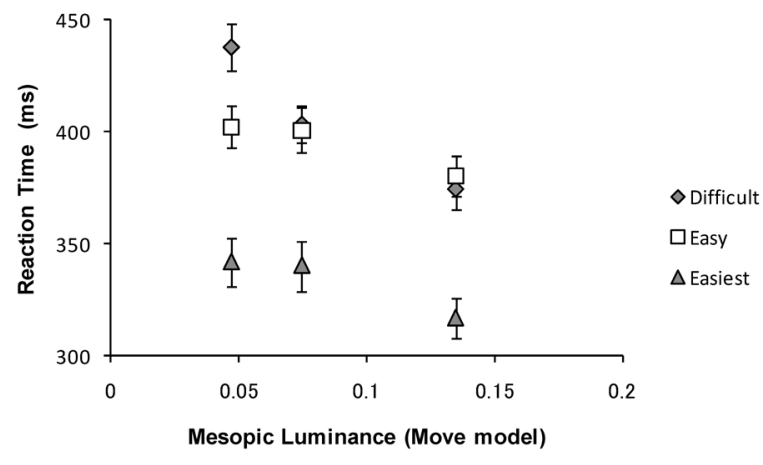

(b)

Figure 7 Comparison of mesopic luminance between (a) USP and (b) MOVE. Also shown are errors under different lighting conditions. Reaction times were averaged across all subjects deviation than those under the other conditions for each of the two mesopic photometry systems. This suggests that it is important for researchers to employ a simple easy task in order to establish a database and a photometry model so that any task interference cannot be involved in it.

\section{Conclusions}

The experimental results generally reinforce many studies, in which mesopic luminance is a suitable rectifying variable for characterizing peripheral visual performance under different light levels and different light sources. The results also confirmed that the use of light sources with more short-wavelength radiation is effective to improve the visibility in lit streets. However, the results also suggested that photopic luminance was an appropriate way to represent the effectiveness of light for foveal tasks. Therefore, even if shortwavelength enriched lamps are applied for roadway lighting applications, it is dangerous to reduce illuminance recommendations for foveal driving tasks.

We also found interference between the foveal task and the peripheral task (especially when the foveal task was difficult). Such interference not only impairs the foveal task performance but also reduces the peripheral responses significantly, and therefore the Purkinje phenomena appeared to be exaggerated. This confirmed the importance of using light sources with more shortwavelength radiation for better peripheral visibility in nighttime roadway lighting applications. Such interference also suggests that the use of a simple easy task may help eliminate any effect of interference on databases to establish a unified mesopic photometry system.

\section{Acknowledgments}

This paper was based on an experiment which we conducted at the Lighting Research Center, Rensselaer Polytechnic Institute. We would like to acknowledge Mark Rea, John Bullough, John Van Derlofske, and Mariana Figueiro, Nick Skinner, Richard Pysar, and Howard Ohlhous for their useful advices and technical contributions to the experiment.

\section{References}

(1) Rea, M. S., Bullough, J. D., Freyssinier, J. P. and Bierman, A.: A proposed Unified System of Photometry, Light. Res. Technol., 36(2), pp. 85-111 (2004).

(2) Eloholma, M., Viikari, M., Halonen, L., Walkey, H., Goodman, T., Alferdinck, J., Freiding, A., Bodrogi and V'arady, G.: Mesopic models-from brightness matching to visual performance in night-time driving: A review, Light. Res. Technol., 37(2), pp. 155- 
175 (2005).

(3) Commission Intemationale de l'Eclairage. CIE 191: Recommended System for Mesopic Photometry Based on Visual Performance, Vienna: CIE (2010).

(4) Goodman, T., Forbes, A., Walkey, H., Elholma, M., Halonen, L., Alferdinck, J., Freiding, A., Bodrogi, P., Varady, G. and Szalmas, A.: Mesopic visual efficiency IV: A model with relevance to night-time driving and other applications, Light. Res. Technol., 39(4), pp. 365-392 (2007).

(5) Bullough, J. D. and Rea, M. S.: Simulated driving performance and peripheral detection at mesopic and low photopic light levels, Light. Res. Technol., 32, pp. 194-198 (2000).

(6) Lingard, R. and Rea, M. S.: Off-axis detection at mesopic light levels in a driving context, J. Illum. Eng. Soc., 31, pp. 33-39 (2002).

(7) Akashi, Y. and Neches, J.: The effects of task load on peripheral target detection, Proceedings of the Commission Internationale de l'áclairage, 25th Session, Commission Internationale de l'áclairage, D4/44-D4/47 (2003).

(8) Akashi, Y.: Research Matters: Attending to attention, Light. Design Appli., 35(12), pp. 10-12 (2005).

(9) Verwey, W. B.: How can we prevent overload of the driver? In Oarkes A. M. Franzen S., Driving Future Vehicles, London: Taylor \& Francis, pp. 235-244 (1993).

(10) Harms, L.: Drivers' attentional responses to environmental variation: A dual-task real traffic study, In Gale A. G. et al., Vision in Vehicles II, Amsterdam: Elsevier Science Publishers, pp. 131-138 (1986).

(11) Pashler, H. E.: The Psychology of Attention, Cambridge, MA: MIT press (1998).

(12) Johnson, A. and Proctor, R. W.: Attention: Theory and Practice, Thousand Oaks, CA: Sage Publications, Inc., pp. 30-37 (2004).

(13) Welford, A. T.: The psychological refractory period and the timing of high speed performance-A review and a theory, Brit. J. Psychol., 43, pp. 2-19 (1952).

(14) Vince, M.: Rapid response sequences and the psychological refractory period, Brit. J. Psychol., 40, pp. 23-40 (1949).

(15) Navon, D. and Miller, J.: Queuing or sharing? A critical evaluation of the single-bottleneck notion, Cogn. Psychol., 44, pp. 193-251 (2002).

(16) Curcio, C. A., Sloan, K. R., Kalina, R. E. and Hendrickson, A. E.: Human photoreceptor topography, J. Comp. Neurol., 292: pp. 497-523 (1990).

\section{Appendix}

The equation that calculates unified luminance $(\mathrm{L})^{1)}$ is

$$
\mathrm{L}=\left[\frac{X P}{683}+\frac{(1-X) S}{1700}\right] \frac{683}{X+0.402(1-X)}
$$

where $P$ is the photopic luminance in $\mathrm{cd} / \mathrm{m}^{2} . S$ is the scotopic luminance in $\mathrm{cd} / \mathrm{m}^{2}$.

The relationship between $L$ and $X$ can be modelled as a linear function between 0.001 and $0.6 \mathrm{~cd} / \mathrm{m}^{2}$, written as:

$$
X=1.669 L-0.001669
$$

The unified luminance $L$ can be expressed as a solution to the following quadratic equation.

$$
\begin{aligned}
L= & 0.834 P-0.335 S-0.2 s \\
& +\sqrt{0.696 P^{2}-0.333 P-0.56 P S+0.113 S^{2}+0.537 S+0.44}
\end{aligned}
$$

If $L>0.6$, then $L=P$. If $L<0.001$, then $L=S$.

The authors presented part of this work at the 39th annual conference of the Illuminating Engineering Institute of Japan, Osaka, Japan in August 2005. Part of this work is published on Lighting Design and Application, Vol. 36, No. 9, 18-20 (2006). 